\title{
Letter
}

\section{Modified Technique for Lengthy, Curved, Zigzag Running Subcuticular with Nonabsorbable Suture}

\author{
Samer Makki Mohamed Al-Hakkak \\ Department of Surgery, Faculty of Medicine, Jabir Ibn Hayyan Medical University, Najaf City, Iraq \\ Email address: \\ Sammerhakak1971@yahoo.com, sammerhakak1971@gmail.com
}

\section{To cite this article:}

Samer Makki Mohamed Al-Hakkak. Modified Technique for Lengthy, Curved, Zigzag Running Subcuticular with Nonabsorbable Suture. Journal of Surgery. Vol. 6, No.1, 2018, pp. 8-12. doi: 10.11648/j.js.20180601.12

Received: November 27, 2017; Accepted: December 8, 2017; Published: January 5, 2018

\begin{abstract}
The use of non-absorbable sutures rather than embedded absorbable sutures in running subcuticular technique is preferable. The reasoning is that buried knot placement and embedded suture material under the skin increases the risk of granuloma formation, ugly scarring, and infection. Removal of the suture material reduces these risks. The new modified technique adopted for easily removed of non-absorbable monofilament using in long, curved and zigzag running subcuticular suturing by put interrupted rubber or plastic tube in the special technique that helps to get easily removed sutures with wonderful, scarless, best cosmetic suture line. Technical considerations go into placing these multiple pieces of tubes along the sutures line in an easy-to-remove manner.
\end{abstract}

Keywords: Lengthy Running Subcuticular Suture, Nonabsorbable Sutures, Removal, Suture Techniques

\section{Introduction}

A subcuticular suture refers to the more difficult technique of repairing a deep wound that extends below the top layer of skin, or epidermis. It is also called a sub dermal, or subcutaneous suture. For surgeons, suturing is considered an indispensable skill subcuticular suturing is a standard technique in dermatologic surgery. It is an ideal option for closing wounds that have minimal tension and mobility. [1] Suture marks are avoided by running the needle along the skin within the sub-epidermal plane. [2] Running subcuticular sutures are considered to be the "holy grail" of suturing techniques by many. That is to say, when done correctly, they give the best cosmetic outcome of closure. Because the epidermis is penetrated only at the start and end of the suture line, the subcuticular suture effectively eliminates the risk of suture marks. The characteristic feature of this technique is that the suture is placed intradermally. There is no visible evidence of the suture on the epidermal surface. [3] The benefit of running subcuticular suture is the minimal epidermal puncture points allowing the suture to be left in place longer without suture-track scarring. Sometimes, the suture cannot be easily removed, which can lead to a pulling force exceeding the tear strength of the wound. This can result in a ruptured thread that is trapped in the wound, for which several causes exist. It can happen when the thread is too large or varying in size or curved or zigzag. Another possible reason is that the stitches are not parallel to the wound edges or are lying too superficially in the epidermis. Especially in long wounds, this can lead to an immovable suture while being withdrawn in the manner described above. [4] So to prevent the suture ruptures, we use helpful techniques in removing it. Our favorite is to put into the middle of the wound or after about more than 5-10 cm distance of the beginning of running subcuticular put a bridge- like (piece of tube). A removal is necessary due to the risk of tissue reactivity, suture granuloma formation, and the possibility of the suture migrating through the epidermis. [5]

\section{Technique}

We present a simple modified technique for the running subcuticular suture that facilities suture removal when approximating wound edges using non-absorbable nylon or polypropylene sutures especially for long, curved and with sharp corners. 
a) The needle is inserted at the far right corner of the wound, parallel to the incision line, beginning approximately $2-5 \mathrm{~mm}$ rom the apex. The needle is passed from this point, which is lateral to the incision apex, directly through the epidermis, exiting into the interior of the wound just medial to the apex. Note that this first pass may be finessed depending on the technique used or finishing the closure.

b) The tail of the suture material resting lateral to the incision apex and outside the wound, the wound edge is gently reflected back and the needle is inserted into the dermis on the far edge of the wound with a trajectory running parallel to the incision line. The needle, and there ore the suture, should pass through the dermis at a uniform depth. Bite size is dependent on needle size, though in order to minimize the risk of necrosis it may be prudent to restrict the size of each bite. The needle should exit the dermis at a point equidistant from the cut edge from where it entered.

c) The needle is then grasped with the surgical pickups and simultaneously released by the hand holding the needle driver. As the needle is reed rom the tissue with the pickups, the needle is grasped again by the needle driver in an appropriate position to repeat the above step on the contralateral edge of the incised wound edge.

d) A small amount o suture material is pulled through, the skin o the contralateral wound edge is reflected back, and the needle is inserted into the dermis on the contralateral side o the incised wound edge and the same movement is repeated. The needle should enter slightly proximal (relative to the wound apex where the suture line began) to the exit point, thus introducing a small degree of backtracking to the snake-like flow of the suture material. This will help reduce the risk of tissue bunching.

e) The same technique is repeated on the contralateral side until the end o the wound is reached. At this point, the needle is inserted from the interior of the wound in line with the incision line and exits just lateral to the apex of the wound (Figures 1 and 2)

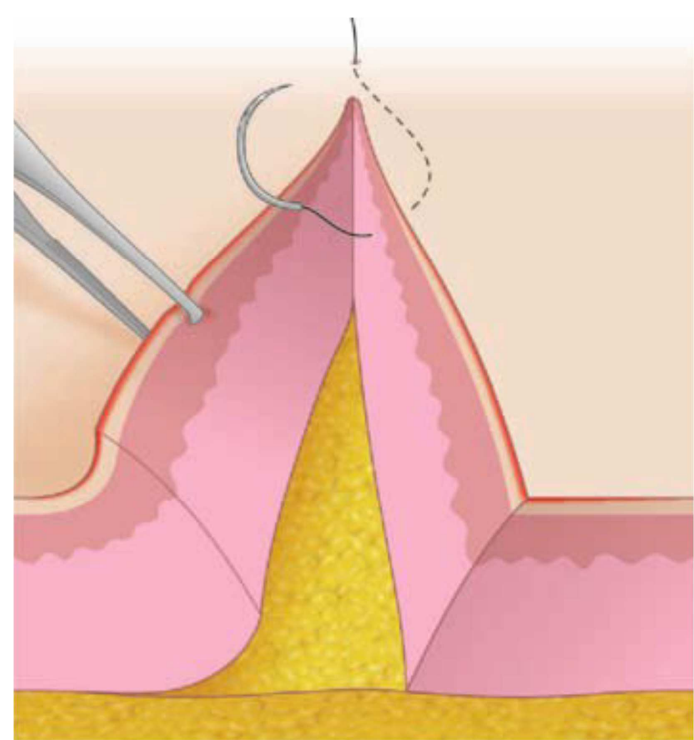

Figure 1. After passing the needle from outside of the wound apex to the interior o the wound, the needle is rotated superficially through the dermis.

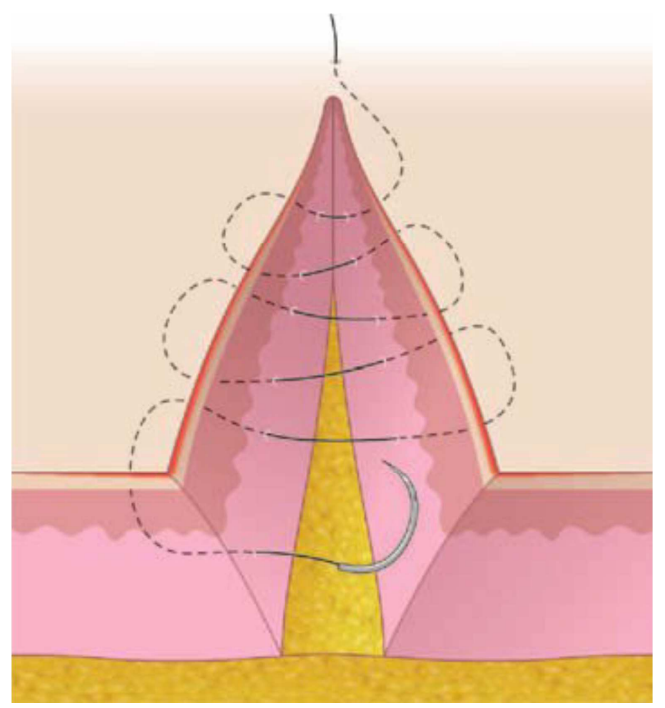

Figure 2. Overview of the running subcuticular technique.

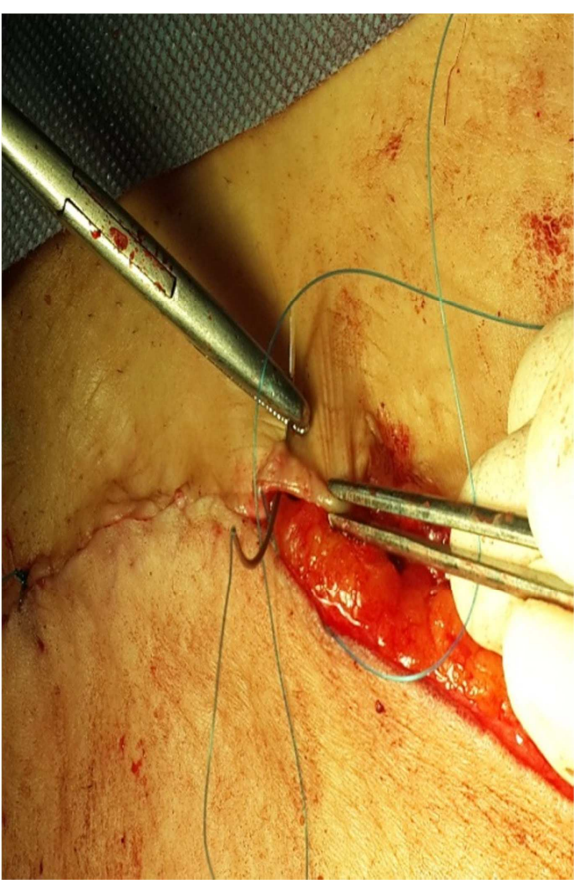

Figure 3. How to pass from inside about $5 \mathrm{~mm}$.

Our technique can be accomplished by placing small piece tube (piece of intravenous line set or piece of small tube from small nasogastric or small tube drain) after every $5-10 \mathrm{~cm}$ distance running subcuticular sutures and continuing this pattern throughout the incision line or sometimes put it in sharp angle of running subcuticular until finishing. We start as ordinary running subcuticular nonabsorbable monofilament nylon or polypropylene is inserted approximately $5 \mathrm{~mm}$ from one end of the wound, horizontal bites of the deep epidermis are taken, and the needle is then passed through the epidermis, then continue in same manner until we reach about $5-10 \mathrm{~cm}$ distance from beginning then we get out from $5 \mathrm{~mm}$ of edge of epidermis (figure 3,4 and 5), introduce piece of tube that equally to the length of each running subcuticular suture and another, after that return back to 
enter the epidermis again and continue the running subcuticular suture line and so on (figure 6 and 7). At the time of suture removal, simply cut the tube then pull from the free ends of the running subcuticular throws of the suture it can be pulled what the direction that needed, which become more visible after cutting the tube. (Figure 8 and 9) This technique makes stitch removal more comfortable for the patient and can be performed more quickly by the medical staff due to relatively easy access to the simple tube and without any scar marking. Thus, the incorporation of the piece of tube inevitably avoids unnecessary trauma during suture removal, especially in patients those who have poor wound healing and those that scar easily.

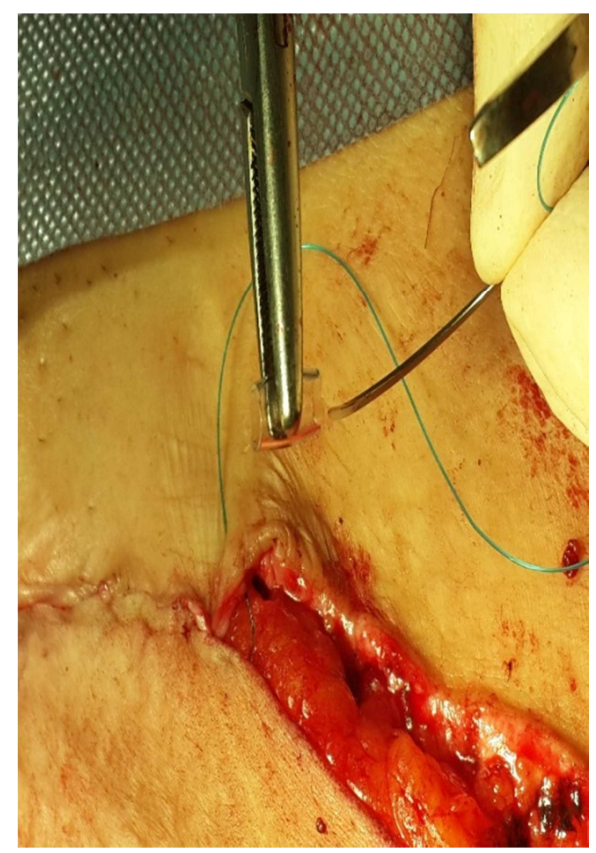

Figure 4. How to try pass through the tube.

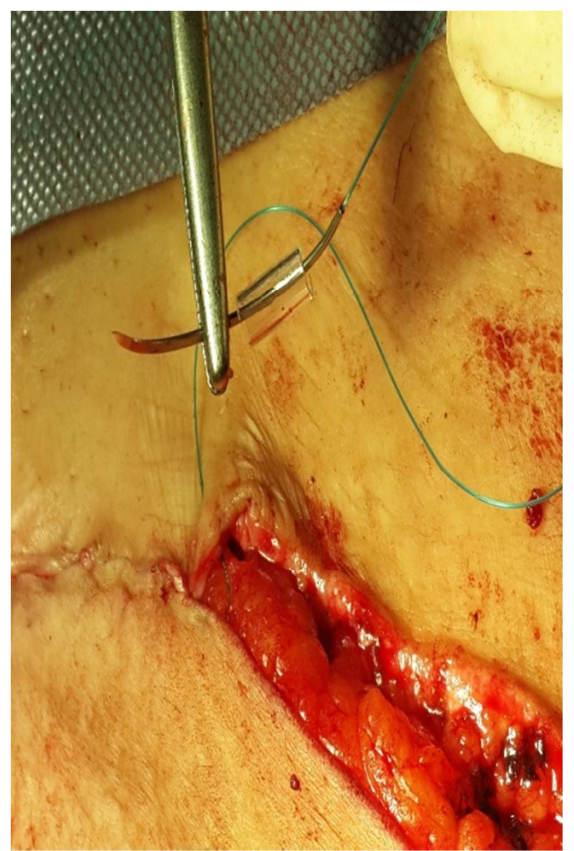

Figure 5. Complelet pass through the tube.

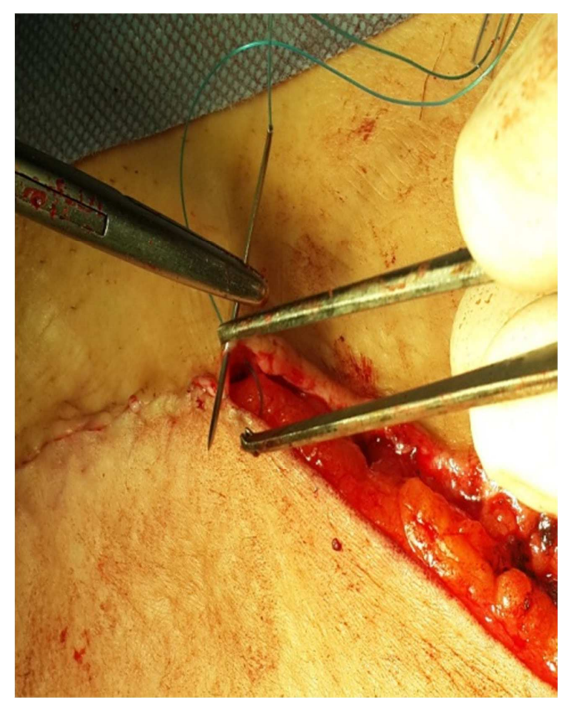

Figure 6. Return back to enter dermis.

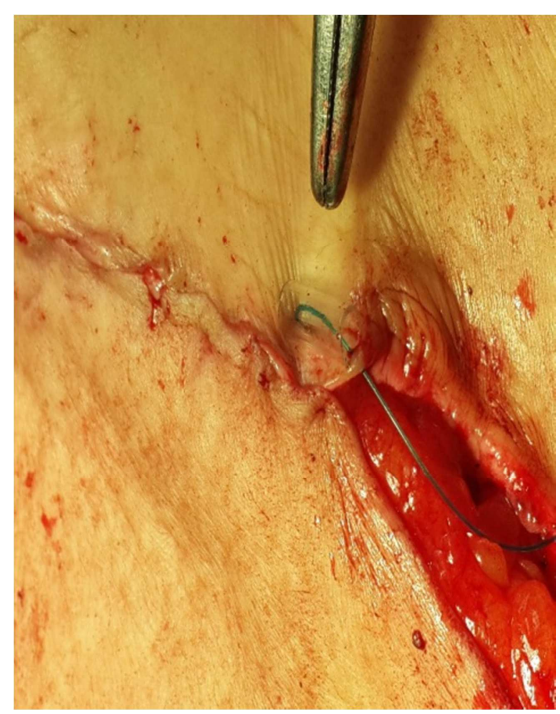

Figure 7. Complete fit the tube.

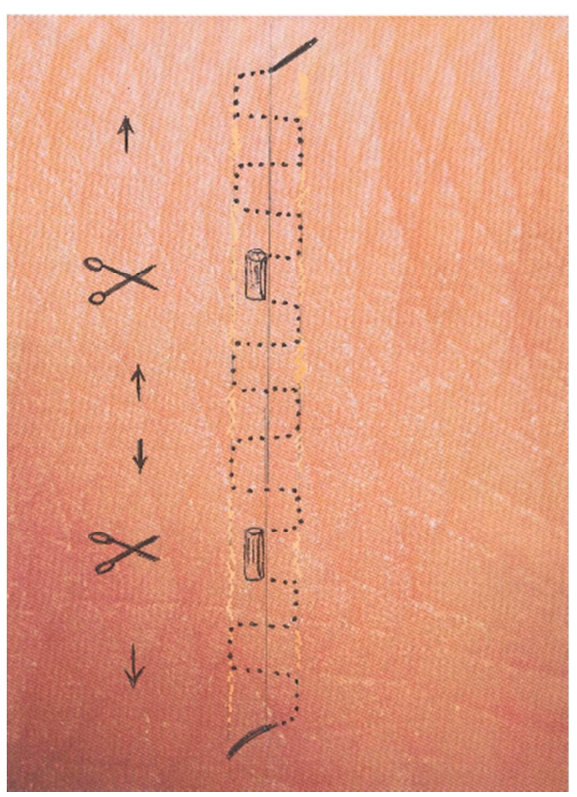

Figure 8. How to remove suture. 


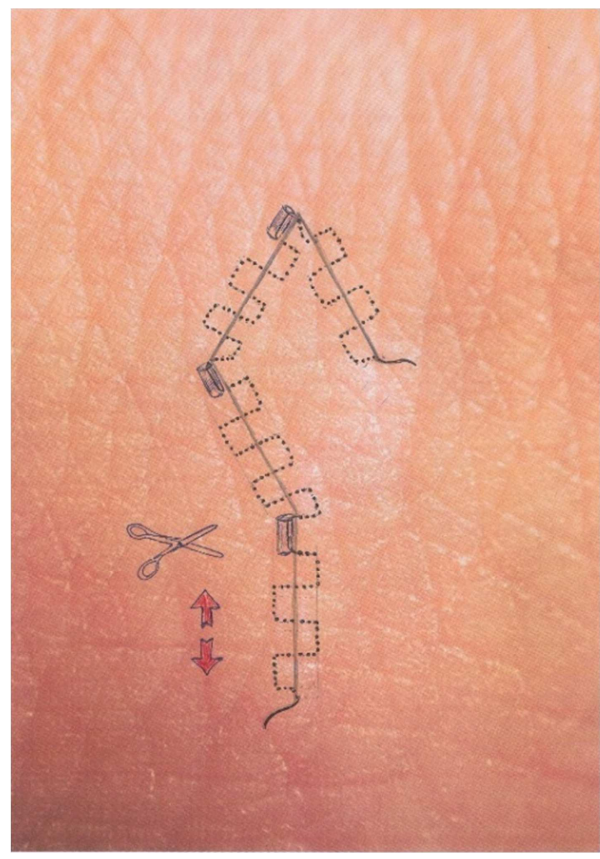

Figure 9. How to remove in rhomboid flap.

\section{Discussion}

This is an epidermal approximation technique suitable or wounds under minimal to no tension. This technique should almost never be employed in the absence of a deep dermal suture, since its strength is in fine-tuning epidermal approximation and it is less effective in the presence of significant tension [6]. Its use is also predicated on the presence of a relatively robust dermis, since it is a primarily intradermal technique and therefore does not recruit any strength from the epidermis. [7, 8] As one of the benefit is to this approach is to permit the suture to remain in place or many weeks or months, it also means that removal of long-standing suture entails a theoretical risk that a potential space - albeit a thin and long one - is created on the removal of the suture material. [9] If nonabsorbable suture material is used, care should be taken to account or the eventual necessity of suture removal. [10, 11] Since the nonabsorbable monofilament will be removed by applying a continuous gentle pull on the free edge of the suture, it is imperative that the length of the continuous suture be kept to a reasonable maximum, to avoid the risk of suture snapping mid pull. In cases where a longer, curved zigzag wound is closed using nonabsorbable suture material, a single simple interrupted tube may be placed as part of the course of the suture material every $4-5 \mathrm{~cm}$ or so, providing a site where the suture material may be snipped and pulled through at the time of suture removal. $[12,13]$

\section{Conclusion}

Using running subcuticular technique with nonabsorbable suture poses the potential risk of wound trauma during suture removal because of embedment. We present a simple modified technique that facilitates suture removal when approximating wound edges with a running subcuticular suture using nonabsorbable sutures. In summary, the combination of running subcuticular suture with a piece of tube demonstrates functional and cosmetic benefits that can be readily taught, comprehended, and employed, ultimately leading to desirable aesthetic results and successful wound without suture marking. It is recommended for all surgical skin sutures especially, long, curved and zigzag line. This technique can be used for easy removal of suture material from the wound without any disruption. This suture technique and removal inevitably reduces wound trauma from suture removal, especially in instances in which the running subcuticular may have been placed with overt backtracking. Obviously, a precise suture technique can prevent potential problems in removing the suture. In lengthy, curved, zigzag wounds which accomplished by running subcuticular suture should have bridges (a small piece of tubes) brought out parallel to the wound, facilitating suture removal. Obviously, a precise suture technique can prevent potential problems in removing the suture.

\section{Conflicts of Interest}

No any conflict of interest.

\section{Funding}

The article receive no funding.

\section{References}

[1] Alam M, Posten W, Martini MC, Wrone DA, et al. Aesthetic and functional efficacy of subcuticular running epidermal closures of the trunk and extremity: a rater-blinded randomized control trial. Arch Dermatol 2006; 142: 1272-8.

[2] Thirumaran M, Jackson A. Suture granuloma. Postgrad Med J 2004; 80: 18 .

[3] Adams B, Levy R, Rademaker AE, Goldberg LH, Alam M. Frequency of use of suturing and repair techniques preferred by dermatologic surgeons. Dermatol Surg. 2006; 32: 682-9. [PubMed].

[4] Tantawy, HS New technique for subcuticular suture in lengthy wounds. Br J Surg 1996; 83: 66-7.

[5] Skaria AM. The Buried running dermal subcutaneous suture technique with a tacking knot. Dermatol Surg 2002; 28: 73941.

[6] Figueroa D, Jauk VC, Szychowski JM, et al. Surgical staples compared with subcuticular suture or skin closure a ter cesarean delivery: a randomized controlled trial. Obstet Gynecol. 2013; 121 (1): 33-38. Genders RE, Hamminga EA, Kukutsch NA. Securing the subcuticular running suture. Dermatol Surg. 2012; 38 (10): 1722-1724.

[7] Ahmed AM, Orengo I. Surgical pearl: alternate method of loading needle to facilitate subcuticular suturing. J Am Acad Dermatol. 2007; 56 (5 suppl): S105-106. 
[8] Bickel KD, Gibbs NF, Cunningham BB. The subcuticular "spider" stitch: a simple solution to suture breakage and patient discom ort in long incisions. Pediatr Dermatol. 1998; 15 (6): 480-481.

[9] Clay FS, Walsh CA, Walsh SR. Staples vs subcuticular sutures or skin closure at cesarean delivery: a metaanalysis o randomized controlled trials. Am J Obstet Gynecol. 2011; 204 (5): 378-383.

[10] McKinley LH, Dorton DW. Modif ed intermediate running subcuticular technique with nonabsorbable suture. Dermatol Surg. 2012; 38 (6): 924-925.
[11] Genders RE, Hamminga EA, Kukutsch NA. Securing the subcuticular running suture. Dermatol Surg. 2012; 38 (10): $1722-1724$.

[12] Lazar HL, McCann J, Fitzgerald CA, Cabral HJ. Adhesive strips versus subcuticular suture or mediansternotomy wound closure. J Card Surg. 2011; 26 (4): 344-347.

[13] Mashhadi SA, Loh CY. A knotless method o securing the subcuticular suture. Aesthet Surg J. 2011; 31 (5): 594-595. 